\title{
Itinerarios generizados en Formación Profesional Básica. Resultados de un estudio en centros educativos valencianos
}

\author{
Gendered Itineraries in Basic Vocational Training Findings \\ of a Study in Valencian High Schools
}

ALIÍCIA VILLAR-AGUILÉS (Universitat de València) y SANDRA OBIOL-FRANCÉS (Universitat de València)

Artículo recibido: 6 de mayo de 2020

Solicitud de revisión: 3 de junio de 2020

Artículo aceptado: 2 de septiembre de 2020

Villar-Aguilés, Alícia y Obiol-Francés, Sandra (2021). Itinerarios generizados en Formación Profesional Básica. Resultados de un estudio en centros educativos valencianos.

Recerca. Revista de Pensament i Análisi, 26(1), pp. 155-178. doi:

http://dx.doi.org/10.6035/Recerca.2021.26.1.8

\section{Resumen}

El objetivo del artículo es analizar la Formación Profesional Básica para comprender mejor la significativa segregación por sexo que los datos muestran, tanto en su acceso como en las especialidades cursadas, a partir del análisis de estadísticas educativas. Este análisis se complementa con el uso de metodología cualitativa a través de entrevistas semiestructuradas a 35 estudiantes de FPB procedentes de cinco centros educativos valencianos, comunidad autónoma con una importante implantación de la FPB. Teóricamente partimos de la consideración de Acker (1990) de instituciones educativas como organizaciones con marca de género que contribuyen a construir modelos de masculinidad y feminidad hegemónicos (Connell, 2001). Los resultados muestran la solidez con la que todavía se identifican estereotipos de género que hacen persistir lo que denominamos itinerarios generizados, los cuales tienen su incidencia en las decisiones educativas y en las futuras transiciones laborales.

Palabras clave: formación profesional, género, alumnado, trayectorias educativas, investigación cualitativa.

\section{Abstract}

The aim of the article is to analyse the Basic Vocational Training in order to better understand the significant segregation by sex that the data show, both in their access and in the specialties studied, based on the analysis of educational statistics. This analysis is comple- 
mented by the use of qualitative methodology through semi-structured interviews with 35 BVT students from five Valencian educational centres, an autonomous region with an important implementation of BVT. Theoretically, we start from Acker's (1990) consideration of educational institutions as organizations with a gender mark that contribute to building hegemonic models of masculinity and femininity (Connell, 2001). The findings show the robustness of gender stereotypes are still identified that persist what we call gendered itineraries, which have an impact on educational decisions and future job transitions.

Key words: vocational training, gender, students, educational pathways, qualitative research.

\section{INTRODUCCIÓN}

Resulta poco cuestionable afirmar que, en general, los estudios de Formación Profesional en el Estado español están fuertemente masculinizados. Las cifras son claras al respecto. En el curso 2017-2018 un 54,63\% del alumnado matriculado en Formación Profesional de Grado Superior eran chicos, un $58,13 \%$ en el caso del Grado Medio y un 70,76\% en Formación Profesional Básica. ${ }^{1}$ Se trata de cifras que reclaman, a nuestro entender, de una mirada a la Formación Profesional desde el binomio sexo/género y su capacidad explicativa para la realidad educativa y, estrechamente relacionada con esta, la realidad laboral. En este sentido, como ya hemos llevado a cabo en otros ciclos educativos (Villar, 2017b), son necesarios análisis desde una perspectiva de género, es decir, tratando de identificar cuál es el peso del sexo/género en la construcción de los itinerarios formativos (y laborales) de los y las jóvenes.

Cuando nos referimos a aplicar una perspectiva de género no significa únicamente desagregar los datos y discursos sobre hombres y mujeres, obviamente, ni referirnos únicamente a mujeres o a aquello que entendemos por sexo (Villar, Grau i Obiol, 2014), pero es una primera forma para aproximarnos a algo tan complejo como es el género y, al mismo tiempo, tan necesario de abordar para analizar la realidad social.

Datos procedentes del Ministerio de Educación y Formación Profesional: Enseñanzas no universitarias. Formación Profesional. Curso 2017-2018. Recuperado de: http://www.educacionyfp.gob.es/servicios-alciudadano/estadisticas/no-universitaria/alumnado/formacion-profesional/2017-2018.html [Consultado en abril de 2020]. 
Este artículo presenta parte de los resultados del análisis realizado sobre la segregación por sexo que existe en la FPB. ${ }^{2}$ Son dos las razones que nos conducen a este propósito. En primer lugar, el hecho de que el acceso a la FPB es vehiculado a partir de una propuesta realizada por los y las docentes a las madres, padres o tutores legales, ${ }^{3}$ pues no podemos obviar que la FPB se encuentra integrada en la etapa obligatoria de la Educación Secundaria. Este hecho hace muy interesante el estudio de la FPB desde una perspectiva de género, puesto que puede contribuir a conocer mejor cómo se construyen las identidades de género y los estereotipos que las sustentan, y cómo estas se reproducen al optar, o no, por FPB, pero también por una familia profesional en concreto. Estos estereotipos forman parte de nuestra socialización y se reproducen entre el alumnado, profesorado, familias, medios de comunicación e instituciones públicas de forma que dan continuidad a la segregación formativa y ocupacional (Cabrera, 1996; Frutos, 2010; Martínez García y Merino, 2011; Fernández e Ibáñez, 2018; Vázquez-Cupeiro, 2015). Y continúan siendo las ocupaciones fuertemente feminizadas las que obtienen un menor valor material y simbólico (Barberà, Candela y Ramos, 2008; Vergés, 2012).

En segundo lugar, asumir como objetivo de investigación analizar la FPB, un ciclo formativo que se implanta en el curso 2014-2015 como una medida de lucha contra el abandono educativo integrado en la Ley Orgánica 8/2013, para la Mejora de la Calidad Educativa (LOMCE), sustituyendo a los anteriores Programas de Cualificación Profesional Inicial (PCPI). Se abre una vía en la educación obligatoria, con un carácter más práctico y orientado a adquirir destrezas de carácter profesional, para aquel alumnado en riesgo de desenganche del sistema educativo para evitar su abandono. Es una vía claramente masculinizada. Por ello nuestro interés es aplicar en su análisis una mirada donde el sexo/género sea relevante. Los datos en este sentido corroboran la pertinencia del análisis, en primer lugar, por la transcendencia del fenómeno, pues el Estado español se encuentra a la cabeza de los países de la Unión Europea en cifras de abandono educativo temprano con un $17,9 \%$ (Eurostat, 2018) que, sin embargo, no se distribuyen de manera equitativa por sexo: un $21,4 \%$ de los hombres jóvenes abandonan los estudios frente al $13 \%$ de las mujeres (Ministerio de

\footnotetext{
${ }^{2}$ Este artículo parte de una investigación más amplia. La investigación que da origen a este artículo fue financiada por el Centro Reina Sofía de Adolescencia y Juventud en su convocatoria del 2018 y el equipo investigador estuvo formado por: Sandra Obiol (IP), Elisabet Almeda, Dino Di Nella, Nuria Pumar, Aida Ruiz, Núria Vergés y Alícia Villar.

${ }^{3}$ Real Decreto 127/2014, de 28 de febrero, BOE, núm. 55 de 5 de marzo de 2014.
} 
Educación, 2019). A nuestro entender, la elevada masculinización del fenómeno contribuye a una invisibilización de las especificidades que los diferentes procesos de desenganche educativo pueden presentar entre chicos y chicas (Jackson, 2006; Jackson y Tinkler, 2007), especialmente cuando el discurso mayoritario en el ámbito mediático y político se centra en el éxito educativo de las mujeres y no en los existentes casos de fracaso. Consideramos importante profundizar en las percepciones y los significados que chicas y chicos dan a su tránsito hacia una FPB puesto que puede mejorar nuestro conocimiento sobre su realidad y, por tanto, aportar mejores bases para la implementación de medidas que vengan a darle solución.

Es por eso que nuestra propuesta reclama una imprescindible perspectiva de género en el análisis de los itinerarios educativos, así como del abandono temprano de los estudios. En este caso focalizamos en la FPB a través de la segregación por sexo en familias profesionales. Hemos centrado nuestra investigación en el caso valenciano, segunda comunidad autónoma con mayor número de alumnado matriculado en FPB en el Estado español, un 15,39\%, únicamente superado por Andalucía con un 17,58 \% en el curso 2017-2018. Esta misma concentración también se da cuando nos referimos a los centros educativos, representando los valencianos el $16,39 \%$ del total de centros que imparten estas enseñanzas, únicamente superado por el 19,93\% de Andalucía. ${ }^{4}$

Comenzamos nuestro artículo con un marco teórico que pivota en la idea central de la existencia de itinerarios generizados. A continuación, pasamos a exponer nuestros resultados, previamente acotados con las decisiones metodológicas tomadas en nuestro estudio: una aproximación a los itinerarios de la FPB mediante un recorrido por estadísticas educativas concretando en el caso valenciano y los resultados del análisis realizado de entrevistas a estudiantado de FPB en centros educativos valencianos. Finalizamos con unas conclusiones en las que subrayamos la necesidad de incluir en el análisis el eje estructural sexo/género para poder tener un conocimiento más ajustado de cómo se construyen las trayectorias formativas y qué consecuencias pueden tener para la vida de las personas que las transitan.

Datos procedentes del Ministerio de Educación y Formación Profesional. Enseñanzas no universitarias. http://www.educacionyfp.gob.es/servicios-al-ciudadano/estadisticas/no-universitaria.html [Consultado en abril de 2020]. 


\section{TRAYECTORIAS EDUCATIVAS Y GÉNERO: ITINERARIOS GENERIZADOS}

Aunque la igualdad es en términos formales un hecho y un logro consolidado en las últimas décadas, existen procesos paralelos a esta igualdad formal que cuestionan la igualdad real y generan segregaciones de tipo vertical y horizontal, tal y como han puesto de relieve algunas voces en el estudio de las desigualdades de género en la educación (García de León y García de Cortázar, 2001; Guil y Flecha, 2015; González Ramos, 2014). Ante este tipo de segregaciones que continúan produciéndose de manera no formal, en un sentido de no evidentes e incluso no siempre reconocidas, podemos preguntarnos hasta qué punto el sistema educativo en su estructura formal actual está produciendo y reproduciendo esas segregaciones.

Los estereotipos y roles de género tan anclados en nuestra sociedad se encuentran significativamente presentes dibujando itinerarios con marca de género o, como nosotras lo expresamos aquí, itinerarios generizados. Nos referimos a itinerarios generizados entendiéndolos no como itinerarios fijos y sin posibilidad de ser alterados o transformados, sino como trayectorias formativas y profesionales que contienen «códigos de género», tomando aquí la expresión de Madeleine Arnot (2002) y haciendo referencia al «modo de transmisión de las relaciones de género a través de conocimientos y estructuras pedagógicas» (Arnot, 2002: 8), estableciendo una compleja intersección con otros componentes sociológicos como la clase social, la etnicidad o la edad, que dibujan los contornos de la desigualdad. Es decir, un desigual acceso a oportunidades educativas, así como a la manera de vivenciar la escuela. En este sentido, el género sirve de base para la organización de relaciones dentro de la escuela al tiempo que diseña opciones formativas más apropiadas para unos y otras según ideas de masculinidad y de feminidad hegemónicas, tal y como son entendidos estos modelos desde los roles sociales tradicionales. Reproducen itinerarios formativos con un código de género operando como itinerarios generizados.

Además, las instituciones educativas que posibilitan en mayor o menor medida, o en un sentido u otro, opciones y oportunidades pueden ser entendidas como organizaciones generizadas, en el concepto y sentido propuesto por Joan Acker (1990). Esta perspectiva incluye no solo a las organizaciones, sino también a las políticas públicas, las cuales se encuentran también inclinadas por los valores atribuidos a aquello que se entiende culturalmente como masculino y femenino. 
Acker expresa de la siguiente manera lo que puede entenderse por organizaciones generizadas: «Afirmar que una organización, o cualquier otra unidad analítica, está generizada significa que las ventajas y las desventajas, la explotación y el control, la acción y la emoción, los significados y las identidades, cobran forma a través, y en términos de una distinción entre varón y mujer, y entre lo masculino y femenino» (Acker, 1990: 146). Y remarca la necesidad de analizar el género en los procesos y las organizaciones, que podemos trasladar también al ámbito de las políticas públicas. Dice Acker: «El género no es un proceso que se añade a otros ya en curso concebidos como neutrales. Por el contrario, es una parte integral de estos procesos que no pueden ser adecuadamente comprendidos sin un análisis de género» (Acker, 1990: 146). Desde el feminismo «el género ha sido elaborado como un concepto que significa más que una identidad e imagen binaria construida socialmente» (Acker, 1990: 145).

Así pues, las instituciones educativas pueden analizarse como organizaciones generizadas que contienen unos códigos de género que funcionan como transmisores, produciendo sesgos y segregaciones de género. Este planteamiento encuentra similitudes con la propuesta de la autora alemana Bettina Dausien, especialista en el estudio de la biograficidad, y que ella expresa como «entornos de género». La biograficidad y el género constituyen un campo de análisis esencial para Dausien porque, como ella afirma, las personas constituyen sus biografías como posicionamientos en el espacio social, un espacio marcado por la relación de género, ya que «el género es una condición estructural, social y del mundo de la vida, de una persistencia considerable y difícilmente hay espacios sociales, sean los que sean, que estén libres de la definición (binaria) de los roles de género» (Alheit y Dausien, 2007: 79).

La propuesta de Dausien se ajusta a la metodología biográfica que utiliza, fundamentalmente, material empírico basado en narrativas. Desde la perspectiva biográfica es esencial entender que la interpretación de las narrativas no busca la emisión de generalizaciones de representatividad estadística, sino comprender el fenómeno social, socioeducativo en este caso, es decir, la comprensión de las decisiones educativas marcadas por el curso biográfico y condicionadas por esos entornos de género que destaca Dausien. Este enfoque biográfico y de género lo hemos aplicado en trabajos precedentes para la comprensión de las trayectorias educativas y las transiciones a la educación posobligatoria (Villar, 2017b).

Esa capacidad organizativa que ejerce el género en las escuelas es desarrollada ampliamente por la socióloga Raewyn Connell (2001) a partir de su teoría 
relacional del género. Se trata de una propuesta teórica para el estudio del género que puede ser considerada como una teoría de la práctica y que aporta una interpretación de la agencia a partir de dimensiones, esencialmente: 1) relaciones de poder, que ejercen tanto docentes sobre su alumnado como entre iguales, aquí Connell apunta que la masculinidad está asociada al poder y la supervisión; 2) relaciones laborales o división del trabajo, que apunta a un mayor número de mujeres en las primeras etapas educativas y en unas determinadas áreas de estudio o asignaturas, así como en el alumnado a la hora de la realización de tareas y vocaciones; 3 ) relaciones de afecto y sexualidad presentes en las escuelas que estarían vinculadas a los regímenes de género que marcan unos límites e imposiciones, como el caso de la homosexualidad; y 4) relaciones simbólicas, que se encuentran enraizadas en las escuelas por su pertenencia a una sociedad en base a una cultura con una simbolización del género, así como un currículum entendido como una estructura simbólica. Estas dimensiones componen lo que denomina Connell órdenes y regímenes de género, siendo el género considerado como relacional, una manera de ordenar la práctica social, y entendido como dinámico siendo las categorías mujeres y hombres frecuentemente tomadas como categorías fijas y sin cuestionamiento.

En la escuela se construye el género como relaciones de poder de género. Connell advierte que, si a las escuelas se las culpabiliza habitualmente de todo tipo de problemas sociales, no nos tendrá que sorprender que también se las culpabilice de modelar las masculinidades/feminidades (Connell, 2001). Añadimos que, en esa construcción social y cultural del género dentro del proceso educativo, el tratamiento histórico desigual de la escuela segregada dejó una impronta histórica y sociológica muy marcada. Como ha elaborado a lo largo de su obra la socióloga catalana Marina Subirats $(1988,2016)$ el sistema educativo ha sido altamente discriminatorio para las mujeres porque no han estado destinadas a los estudios. Posteriormente, el modelo de escuela mixta resultó determinante para el avance educativo de las mujeres, obviamente, pero no ha sido suficiente para poder desterrar todas las discriminaciones. Como propone Subirats, en la escuela actual continúan perpetuándose un tipo de dispositivos de discriminación que ya no son ni patentes, ni reconocidos, que posibilitan lo que denomina el «aprendizaje de subordinación» a través de «la transmisión de la cultura androcéntrica, a través del currículum oficial y del currículum oculto que transmite a las niñas su carácter secundario en el mundo público, su no significancia en él y, por tanto, su no protagonismo» (Subirats, 2016: 29).

En el estudio de las decisiones educativas esta propuesta teórica nos puede permitir analizar cómo determinados itinerarios contienen ese mandato de 
subordinación o, contrariamente, de dominación. Los itinerarios generizados están impregnados de esos aprendizajes y de esos roles sociales o mandatos de género de la sociedad a la que se destinan. Ante las segregaciones y las relaciones de género que perpetúan relaciones de poder no igualitarias y que las instituciones educativas permiten, más o menos conscientemente, el primer paso para su transformación, porque entendemos que son transformables por el sentido dinámico y permutable que apunta Connell, sería reconocer y analizar esas segregaciones de género.

En los últimos tiempos la investigación sobre género y educación está tomando fuerza y es un campo de investigación consolidado. Investigaciones que se atreven con ese «proyecto complejo y dinámico», que advertía Arnot, que es investigar sobre género y educación. También, como expresa Acker y otras investigadoras feministas, consideramos la necesidad de reconocer la complejidad que supone investigar el género, tanto en lo epistemológico, como en el abordaje metodológico y empírico, en un sentido no binario y en su dimensión dinámica y cambiante, puesto que con frecuencia queda reducido a las categorías binarias, especialmente en el caso de la utilización de indicadores educativos. Y, en este trabajo, y en la línea de investigación que desarrollamos, nos situamos en esa perspectiva.

\section{EL GÉNERO EN LOS ITINERARIOS A TRAVÉS DE LAS ESTADÍSTICAS EDUCATIVAS. EL CASO DE LA FORMACIÓN PROFESIONAL BÁSICA}

La perspectiva de género es necesaria e incuestionable a la hora de analizar la evolución de los indicadores educativos. Detrás de decisiones y trayectorias educativas continúan incidiendo los modelos de masculinidad y de feminidad hegemónicos que arrastran a las personas a tomar caminos distintos en sus estudios que condicionan el posterior desarrollo profesional y, con ello, aunque se hayan alcanzado avances, la persistencia de la división sexual del trabajo. En este sentido los datos desagregados por sexo nos muestran cómo hay profesiones claramente masculinizadas y otras claramente feminizadas. Según el informe elaborado por Hardy, Burchell, Rubery y Smith (2015) para el contexto europeo, solamente el $18 \%$ de las mujeres trabajan en ocupaciones mixtas (6o$40 \%$ hombres y mujeres), el $69 \%$ en ocupaciones dominadas por mujeres (> $60 \%$ mujeres) y solo el $13 \%$ en ocupaciones dominadas por hombres (> 60\% hombres). De manera contraria, solo el 15 \% de los empleados varones trabaja- 
ban en ocupaciones mixtas, el $59 \%$ en ocupaciones dominadas por hombres y un $26 \%$ en ocupaciones donde hay un mayor número de mujeres.

En el trasfondo de esa segregación ocupacional se encuentra una segregación anterior, asociada a las decisiones educativas y a la construcción de trayectorias formativas. 5 Si bien en la educación obligatoria (infantil, primaria y ESO) chicos y chicas se distribuyen en una proporción equilibrada, las distancias se hacen más significativas en los itinerarios de la posobligatoria; es el caso del bachillerato presencial, en el que las chicas son ligeramente mayoría (un $54,2 \%$ en el caso valenciano y un 52,7 \% en el estatal). Es una apuesta más clara por parte de las chicas por itinerarios más académicos (Martínez García y Merino, 2011). De hecho, los datos nos muestran una mayor presencia de los chicos en la formación profesional: en los ciclos formativos de grado medio del sistema valenciano ellos son un 56,7\% y ellas son un 43,3\% (en el caso español hay una mayor proporción de hombres, un 57,7\%); en el caso de los ciclos superiores presenciales ellas ascienden a un $47,1 \%$ Y, en el caso que nos ocupa, la FPB, es donde se produce una mayor asimetría entre hombres (69,5\%) y mujeres $(30,5 \%)$, la cual es menor en el caso valenciano que en el español $(71,1 \%$ y $28,9 \%$ ); mientras que en la opción de formación a distancia las mujeres alcanzan una cifra muy elevada, un 60,2 \% (en el caso español es un 59,5\%).

Estas diferencias de matriculación evidencian la existencia de diferencias en la elección de itinerarios. Y todavía es más clara cuando nos sumergimos en el detalle de los datos de matriculación según familias profesionales. ${ }^{6}$ En el curso 2016-2017 y para el caso valenciano, las familias profesionales con mayor peso son, por este orden, Administración y Gestión (21,4\%), Informática y Comunicaciones (18,9\%), Electricidad y Electrónica (14,4\%), Agraria (10,1\%), Imagen Personal (7,1\%), Hostelería y Turismo (5,8 \%), Comercio y Márquetin (5,6\%), Fabricación Mecánica (5,5\%), Transporte y Mantenimiento de Vehículos $(5,3 \%)$. El resto de las familias profesionales presentan un porcentaje de alrededor del $1 \%$.

5 Los datos incluidos a lo largo del texto que se refieren a estadísticas sobre FP proceden de las Estadísticas de la Educación del Ministerio de Educación y Formación Profesional.

6 La oferta de familias profesionales que se incluyen en la FPB son las siguientes: Actividades Físicas y Deportivas, Administración y Gestión, Agraria, Artes Gráficas, Comercio y Marketing, Edificación y Obra Civil, Electricidad y Electrónica, Fabricación Mecánica, Hostelería y Turismo, Imagen Personal, Industrias Alimentarias, Informática y Comunicaciones, Instalación y Mantenimiento, Madera, Mueble y Corcho, Marítimo-Pesquera, Servicios Socioculturales y a la Comunidad, Textil, Confección y Piel, Transporte y Mantenimiento de Vehículos y Vidrio y Cerámica. Estas familias están implantadas casi en su totalidad en el sistema valenciano, solamente dos de ellas (Actividades Físicas y Deportivas y Servicios Socioculturales y a la Comunidad) no se ofertan por el momento. 
Si observamos cómo se distribuye la matrícula de mujeres y hombres en las familias profesionales con mayor peso en el caso valenciano, observamos asimetrías significativas en las distintas opciones formativas de FPB (tabla 1).

Tabla 1. Distribución porcentual de hombres y mujeres en las familias profesionales en la FPB valenciana curso 2016-2017

\begin{tabular}{lll}
\hline Familia profesional & Hombres & Mujeres \\
\hline Agraria & 81,0 & 19,0 \\
Marítimo Pesquera & 93,3 & 6,7 \\
Industrias Alimentarias & 58,2 & 41,8 \\
Imagen Personal & 16,6 & 83,4 \\
Fabricación Mecánica & 95,7 & 4,3 \\
Instalación y Mantenimiento & 91,8 & 8,2 \\
Electricidad y Electrónica & 95,7 & 4,3 \\
Transporte y Mantenimiento de & 98,1 & 1,9 \\
Vehículos & & \\
Edificación y Obra Civil & 87,1 & 12,9 \\
Vidrio y Cerámica & 67,3 & 32,7 \\
Madera & 90,6 & 9,4 \\
Textil & 41,8 & 58,2 \\
Artes Gráficas & 54,7 & 45,3 \\
Informática & 81,1 & 18,9 \\
Administración y Gestión & 47,0 & 53,0 \\
Comercio y Márquetin & 46,0 & 54,0 \\
\hline
\end{tabular}

Fuente: Elaboración propia a partir de datos del Ministerio de Educación y Formación Profesional.

Las familias profesionales que presentan una distribución más equilibrada (6o/40) son Industrias Alimentarias, Textil, Artes Gráficas, Administración y Gestión y Comercio y Márquetin. En cambio, observamos que la mayor parte de las familias presentan una alta asimetría entre hombres y mujeres y que en la mayoría de los casos responden a concentraciones masculinas en más de un $80 \%$. Solamente se invierte este patrón en el caso de Imagen Personal, con un 83,4 \% de mujeres. Aunque Agraria e Informática son dos opciones también 
masculinizas, las mujeres representan casi dos quintas partes de la matrícula (rozando el $20 \%$ ), lo que supone un avance de su presencia en estas familias profesionales, que por ciclos formativos se concentran especialmente en el ciclo de Agro-jardinería y en el de Informática de Oficina.

Estos datos sobre matriculación y graduación nos llevan al resultado de que o bien chicas y chicos siguen eligiendo de manera diferenciada o bien el sistema y los roles de género persistentes los llevan a situarse en itinerarios diferentes, en unos casos más que otros, como se observa por los datos comentados. Ello contribuye a la segregación ocupacional posterior y nos lleva a identificar que, en gran medida, la segregación entre profesiones masculinizadas y feminizadas se produce y reproduce en el sistema educativo.

Concluimos este apartado destacando que los datos desagregados por sexo en la matrícula de la FPB muestran una distribución de hombres y mujeres con una fuerte asimetría en el caso valenciano. Esta asimetría perpetúa estereotipos de género asignados culturalmente a los itinerarios formativos y contribuye a la persistencia de itinerarios masculinizados y feminizados.

\section{ITINERARIOS DE GÉNERO EN LA VOZ DE SUS PROTAGONISTAS}

Como apuntábamos al comienzo, nuestra investigación ha buscado el lugar que el género ocupa en el itinerario que siguen chicas y chicos hasta cursar Formación Profesional Básica y que les hace decidirse por una u otra familia profesional en la Comunidad Valenciana. Para ello, hemos accedido a un total de 7 centros educativos valencianos donde ofertan este tipo de formación con el propósito de comprender las razones por las que estamos ante una formación marcadamente masculinizada, de manera más evidente que otros itinerarios formativos existentes. Estudiar la FPB desde una perspectiva de género nos ha permitido conocer el peso del género en las trayectorias que pueden conducir a un abandono temprano de los estudios y que la FPB viene a intentar enmendar, al tiempo que ver cómo la construcción de la decisión de cursar un itinerario formativo puede condicionar el lugar futuro en la estructura ocupacional. 


\subsection{Aspectos metodológicos}

Este artículo procede de una investigación más amplia en la que hemos tratado de acercarnos a la realidad de la FPB con la intención de conocer el peso del género en la configuración de esa formación profesional. Para ello, hemos trabajado con un total de siete centros educativos, elegidos según tres variables básicas:

1) En primer lugar, centros educativos que ofrecen itinerarios de FPB fuertemente masculinizados y feminizados, con la intención de trasladar a nuestros casos de estudio la segregación que encontramos en las estadísticas.

2) En segundo lugar, el tamaño del hábitat. Procuramos huir de la fuerza centrípeta de la capital, en este caso València, ${ }^{7}$ con un doble objetivo: sopesar el peso del control social en las decisiones basadas en estereotipos de género, que puede ser más rígido en poblaciones de tamaño reducido, al tiempo que poder llegar a valorar otros factores como la escasez de opciones formativas o la dificultad del acceso a los centros que en ciudades grandes se diluye.

3) Y, por último, la titularidad del centro, pública y privada, una variable que nos remite a la capacidad del centro de contar y gestionar recursos económicos y docentes.

El acceso a los centros fue facilitado por la Dirección General de Formación Profesional y Enseñanzas de Régimen Especial de la Conselleria d'Educació, Investigació, Cultura i Esport de la Generalitat Valenciana y por sindicatos, además de contactos personales. Y, de esta muestra de centros educativos, más en concreto de cinco de estos, se derivaron un total de 35 entrevistas a chicas y chicos cuyos resultados son la base de este artículo. ${ }^{8}$ Aunque el trabajo de campo se llevó a cabo entre julio de 2018 y junio de 2019, las entrevistas en profundidad al alumnado de FРB (21 chicos y 14 chicas) se concentraron durante los meses de diciembre de 2018 a febrero de 2019. Pudimos acceder al alumnado a través de sus tutoras/es, quienes nos facilitaron la posibilidad de poder presentar nuestra investigación a los jóvenes. Si querían participar, debían retornarnos un documento de consentimiento de participación en el es-

De hecho, en el curso 2019-2020, València concentra el 11,42\% de los centros que imparten FPB en todo el territorio valenciano.

8 Pero, además, se realizaron entrevistas grupales al equipo directivo, tutores/as, orientadores/as y coordinadores/as de igualdad, entrevistas en profundidad a madres y padres del alumnado de FPB, entrevistas grupales a chicos y chicas cursando 2. de ESO (en el anexo se detalla por centro cada uno de estos elementos). 
tudio firmado por uno de sus progenitores o tutores, puesto que en la mayoría de los casos se trata de menores de edad.

Las entrevistas se realizaron en el mismo centro educativo, en una sala diferente al aula, durante horario lectivo (generalmente en horario de tutorías). El guion constaba de seis grandes apartados: grado de satisfacción con la FPB, descripción del camino que los había llevado hasta una FPB, razones por las que habían optado por la familia profesional que cursaban, diversos aspectos de su vida cotidiana (relaciones familiares y con sus amistades, uso del tiempo...) y proyección de futuro, además de un apartado de variables sociodemográficas de control. Todas las entrevistas fueron grabadas, transcritas y analizadas a través del software MAXQDA a partir de la identificación de códigos de significado. En los siguientes apartados exponemos los resultados extraídos en nuestra investigación.

\subsection{El camino hacia la FPB}

El análisis de las entrevistas realizadas a alumnado de FPB nos muestra, con claridad, la pervivencia de roles de género tradicionales con diferencias marcadas entre lo que les corresponde dedicarse si son chicas o si son chicos. Y, además, la intensidad de estas diferencias no se distribuye igual por género.

En primer lugar, es preciso remarcar una cuestión básica antes de entrar en las narraciones de las chicas y los chicos matriculados en FPB: hay una escasa agencia en la elección de este ciclo formativo y de la especialidad que cursan, a pesar de que una vez iniciado procuran revestirlo, en mayor o menor medida, de condiciones de elección individual como ya hemos visto anteriormente (Obiol, 2017). La estructura social emerge con fuerza. Por un lado, la clase social, dado que nos encontramos mayormente con progenitores con ocupaciones poco cualificadas, pero también con hermanas y hermanos, especialmente hermanos, con una trayectoria de abandono temprano de los estudios o en situaciones muy próximas al mismo. Han llegado hasta aquí porque es la vía más sencilla que conciben para conseguir una certificación académica, que consideran esencial para poder tener un futuro inmediato que pueda escapar de la precariedad. Son conscientes, y así lo verbalizan, del carácter normalizador de los estudios (Horcas López, Bernad i Garcia y Martínez Morales, 2015). Además, la justificación de seguir en los estudios remite constantemente a la experiencia de sus padres en el mercado de trabajo — estudiar para no ser «una mula de carga» (E21), por ejemplo-, poder optar a un futuro mejor que el de sus padres pasa para ellas y ellos irremediablemente por tener una formación, 
la que sea. Es preciso anotar aquí que la clase social es una variable fundamental en el análisis de los procesos de desenganche educativo (Tarabini, Curran, Montes y Parcerisa, 2019).

Obviamente, su trayectoria recorrida hasta el momento no ha facilitado el hecho de obtener esta certificación académica. Son constantes las referencias a sucesivas repeticiones de curso —en algún caso también en primaria-, absentismo, expedientes sancionadores, expulsiones, visitas a las/los trabajadoras/es sociales... Hay también comportamientos disruptivos: insultos al profesorado, romper mobiliario... Aunque existen, son pocos los que asumen este papel entre los estudiantes entrevistados. En conclusión, apatía y falta de capacidad para implicarse en el curso de los estudios de secundaria.

Y también el género se trasluce en cómo ha sido el camino que los ha llevado a la FPB. Los códigos de género (Arnot, 2002) marcan las trayectorias. Unas trayectorias que hacen patente el peso del género: los chicos tienen, nos explican los entrevistados, un comportamiento más explosivo, lo que nos remite a una persistencia de modelos de masculinidad hegemónica (Connell, 2001), que se distingue de comportamientos asociados a una mayor tranquilidad, por expresarlo así, de las chicas, lo que les hace a ellos más visibles por disruptivos en el ritmo que pretenden guardar los/as profesores/as en las aulas y eso les lleva, irremediablemente, a alternativas al curso habitual de los estudios.

También hay algunos profesores que las protegen, se nota a veces. [...] También las chicas no quieren venir [a FPB] porque es como que esto es de machirulo. (E33, chico.) ${ }^{9}$

Sí, repetí 2.. ${ }^{\circ}$, vine a este [centro], vine a $2 .{ }^{\circ}$ también y no repetí porque tenía que pasar

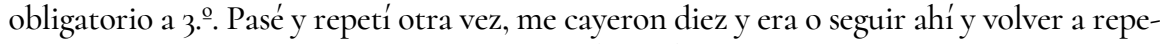
tir, llegar a $4 .^{.} \mathrm{y}$ volver a repetir porque ya te he dicho que no me gusta el sistema, o buscarme un trabajo o FP. (E23, chico.)

Así pues, a la distinción entre «itinerarios de chicos» e «itinerarios de chicas» se suma otra distinción frecuente que está relacionada con el reconocimiento social de los posibles itinerarios formativos: la opción de la formación profesional continúa teniendo un componente de menor reconocimiento y, aunque es visto como probable entre el alumnado de secundaria posobligatoria, es considerado como un «itinerario puente» (para llegar a la universidad) y

9 La mayoría de las entrevistas se realizaron en catalán, lengua materna de muchos de los chicos y chicas entrevistadas. Hemos traducido directamente los extractos utilizados en este texto al castellano para facilitar su lectura. 
como una opción menos prestigiada, según los resultados de una investigación que realizamos en el contexto valenciano sobre transiciones entre la educación secundaria y la educación superior (Villar, 2017a).

\subsection{La FPB como la opción más fácil}

La FPB aparece ante ellos y ellas, porque así se la presentan desde los equipos de orientación de los centros, como una alternativa más factible que la ESO para conseguir una mínima certificación académica al tiempo que una especialidad profesional, que como hemos dicho les resulta muy importante.

¿Te gusta estudiar? No mucho. Aquí me dijeron que era más fácil y los profesores también son... Te tratan más con una relación de igual. (E14, chica.)

A ver, me dijo [la orientadora] que... porque en la ESO ella sabía que no estaba haciendo nada y no quería hacer nada, y me dijo que lo intentara por aquí, que me intentara sacar la ESO por aquí y que me iría mejor aquí. (E16, chico.)

Cierto es que se trata de una elección no demasiado marcada, a veces incluso tomada por otros, por el propio centro nos relatan, y que no les preocupa sobremanera ni parece que hayan invertido mucho tiempo en valorar su pertinencia. Pero sí que valoran de manera positiva la organización de los contenidos con un menor peso de las materias básicas y un mayor peso de materias encaradas al mundo profesional y el contenido importante de horas en prácticas en las empresas, pero valoran especialmente que el profesorado esté muy pendiente de sus necesidades y empatizan con mucha facilidad con el profesorado, puesto que muchos, en especial aquellos que imparten la parte profesional, han tenido trayectorias formativas similares y eso les facilita, según sus palabras, el tránsito por la FPB.

En las entrevistas presentan la FPB como una segunda oportunidad para enmendar decisiones y comportamientos anteriores que, en el momento de la entrevista, consideran totalmente negativos y muestran un arrepentimiento total —en especial aquellos/as que han tenido un comportamiento más disruptivo- de su relación conflictiva con el sistema educativo. 


\subsection{Un camino marcado}

Una vez clara la opción por FРB han de decidir qué especialidad cursar. Y aquí también entendemos con prevención la palabra decisión puesto que las opciones son muy limitadas. La falta de planificación geográfica de los itinerarios junto con su trayectoria académica hace que tengan una menor capacidad de decisión. Un factor importante en la elección son las opciones de proximidad que tienen. No se trata tanto de que estén eligiendo qué quieren hacer, sino qué pueden hacer para continuar integrados en el sistema. Es importante tener esto en cuenta. En los municipios de interior o más pequeños las opciones se ven todavía más limitadas. Hay que pensar que son estudiantes muy jóvenes (16-17 años) y que tienen una capacidad de moverse por el territorio limitada.

Yo antes de meterme aquí quería hacer una FP de cocina, pero me pillaba muy lejos y las becas eran bastante caras, pero al final me metí aquí porque lo tengo a un minuto de mi casa, y tenía todo... [...] (E21, chico.)

Claro, es lo que ha dicho ella, para una básica, nuestras madres no nos van a llevar a [otro pueblo] para hacer una básica, ¿me entiendes? (Eo7, chica.)

Además, en la elección que hacen por optar por la FPB y luego por el itinerario a cursar, las relaciones de amistad son fundamentales, en especial en las chicas, quienes explican con claridad cómo han elegido ciclos porque tenían amigas o conocidas con las que sentirse mejor acogidas. Sin embargo, es habitual que muchos de sus amigos o familiares hayan pasado por opciones similares, como eran los PCPI o el actual PMAR. Las redes de proximidad aparecen con fuerza en cada decisión que explican.

En las conversaciones mantenidas con los jóvenes, a pesar de su frecuente parquedad, nos permiten entrever de manera rotunda la pervivencia de marcos de referencia diferenciados para chicas y para chicos, ese aprendizaje subordinado al que hace referencia Subirats (2016). Los datos ya nos lo hacían evidente, ahora sus palabras también. En primer lugar, resulta importante señalar que únicamente una de las chicas entrevistadas está cursando un módulo masculinizado (Electricidad y Electrónica) y solo uno de los chicos entrevistados, un módulo feminizado (Peluquería y Estética), aunque realizamos entrevistas a la 
mayor parte de estudiantes matriculados en FPB en los centros a los que acu$\operatorname{dimos}^{10}$

¿Cómo lo explican? La mayoría apelando a los gustos o habilidades personales, incluso a la vocación infantil. Claramente estos gustos remiten a una concepción de lo que representa ser mujer y hombre en nuestra sociedad: se trata de itinerarios generizados que se corresponden con unos códigos que funcionan como transmisores de las relaciones y roles de género. Los códigos se encuentran insertos en las recomendaciones del centro educativo, de las orientaciones recibidas por los docentes y orientadores y, cómo no, de códigos forjados más allá del ámbito puramente escolar.

A ellos les gusta mucho y son especialmente hábiles con las maquinarias, en tareas para las que se requiera fuerza y les permitan estar al aire libre, ser autónomos, con los automóviles, no les importa ensuciarse... Ahora bien, se niegan a participar en actividades que supongan contacto físico, que tengan que ver con la imagen física, que supongan limpiar o cuidar de alguna manera.

¿Qué es lo que no te gusta de la peluquería? Es que no sé, estás ahí con la persona, y en su cabeza y... [...] Claro, ¿la silla qué te va a decir? Esto a lo mejor lo reparas con otra pieza que se parezca, pero la cabeza de alguien ¿qué haces? (E27, chico.)

Ellas defienden que su elección por el módulo que cursan ha sido porque les gusta mucho el maquillaje desde niñas, la estética, los cosméticos, cocinar... En absoluto se ven capacitadas para tocar ningún tipo de maquinaria, ni interesadas en realizar redes eléctricas o construir muebles de madera, por ejemplo.

¿Por qué no has hecho carpintería? Porque estética me vuelve loca, te lo juro, me vuelve... [...] Te lo juro. El maquillaje, las uñas, depilar... Todo, me gusta todo, todo, todo. Es que me vuelvo loca, a lo mejor voy a Mercadona y veo todos los maquillajes... Es que me llama, me llama, estoy a gusto haciendo eso y lo hago bien, no sé, me gusta mucho. [...] No sé, es que a mí me nace, te lo juro. (E29, chica.)

No sé... por ejemplo, mecánica, es que yo no me veo en mecánica, montando un coche o un barco, yo no me veo así. (Eo8, chica.)

10 Fueron solo unos pocos los que no aceptaron participar en la investigación y no rompían en absoluto con la diferenciación de género expuesta. 
Y lo explican de manera descarnada, tanto que se puede llegar a pensar que los años de lucha feminista para cambiar los modelos de referencia para las jóvenes no han sido de utilidad. Sin embargo, en cierta medida sí que pensamos que lo han sido, puesto que son ellas las que, aun cumpliendo con este mandato de género, no niegan en absoluto, al contrario, defienden con vehemencia, que chicas y chicos pueden hacer las mismas cosas y están plenamente capacitados para hacerlas y no se intuye de ninguna manera que, en el caso de que decidieran romper con este mandato, sufrirían una sanción como control social (no decimos que no pasaría, sino que ellas defienden que no). Sin embargo, tanto chicos como chicas sí que describen decisiones mucho más condicionadas a la figura masculina hegemónica y con un control social mucho más patente. En ese sentido, las relaciones de poder de género que recorren las instituciones y los procesos educativos, en el sentido que apunta Connell (2001), se expresan claramente en voz del alumnado.

Pues sí, mira, un chico de carpintería le gusta mucho la peluquería y no se apuntó porque éramos todo chicas, porque no había ningún chico. [...] Yo se lo dije, le dije: «¿Por qué no te has apuntado?». Y me dijo: «Porque me daba vergüenza, que me dijeran que podía ser maricón». (E28, chica.)

Yo creo que los chicos tienen miedo de meterse en peluquería porque lo ven más de mujeres. Entonces tienen miedo a que les digan que son afeminados o cualquier cosa de esas, porque ha pasado mogollón de veces. (E31, chica.)

¿Por qué sois todos chicos aquí? Porque ninguna se apunta. [...] Todas a peluquería, que también hay chavales que van a peluquería, pero yo a esos chavales... ¿Qué pasa con esos chavales? Son tíos, pero, pero... Es que, a ver, para decirlo en un tono más delicado, que son [... ] Esos yo creo que serán gays, por decirlo así. Van con chicas, caminan como si fueran chicas, a veces incluso se maquillan y todo, y no, eso no. (Eo1, chico.)

De hecho, la diferencia en las palabras de la chica que rompe con el mandato de género respecto al chico que hace lo mismo es patente: ella no encuentra ningún problema a su alrededor que le lleve a no llevar a cabo lo que quiere y él no hace caso a lo que le puedan decir. Los iguales surgen aquí como un potente elemento de socialización y control social y, tal y como otros autores han identificado, en el caso de los chicos es más grande este control (Julià, 2016).

A ver, el año pasado sí que, en la gente más macarra, por decirlo, que empieza y luego se lo deja, también pensaba que me miraban un poco raro, pero como tampoco los 
conocía... No sé, a mí nadie me dice nada aquí, ni nada es que tampoco me importa que me lo digan. (E33, chico.)

¿Qué te dijeron tus padres sobre el hecho de que estudiaras electricidad? Me dijeron que podía haberme cogido informática o algo así. Me dijeron lo típico de que no era para chicas, pero a mí me da igual; aunque fuese la única chica, a mí me gusta esto, así que me da igual lo que digan. (E14, chica.)

Por cierto, que sus grupos de amigos son fundamentalmente homogéneos respecto el sexo: chicos, si son chicos y chicas, si son chicas. Con pocas excepciones y nos las encontramos, por ejemplo, con el chico que rompe con las expectativas de género de que en su grupo son todas chicas o la chica que hace lo mismo: «Yo de pequeña solía tener más amigos chicos que chicas» (E14).

$\mathrm{Y}$, por último, su futuro laboral y la proyección que hacen ante un mercado de trabajo profundamente segregado por género, además de precario, es fundamental para entender las opciones que toman los y las estudiantes entrevistados. En su caso, la relación con un mercado de trabajo segregado es evidente, sobre todo porque anticipan su acceso al mercado de trabajo desde las redes de proximidad. Con los amigos de los padres que tienen en la hostelería (Eo8, chica) o en la peluquería de la madre (E28, chica) o bien con otros familiares. Incluso padres que tienen a sus hijos haciendo el periodo de prácticas en su taller. Un hecho que no deja de ser significativo por con qué referencias construyen su imaginario respecto a su integración en el mercado de trabajo, a partir de referencias en gran parte de precariedad y muy tradicionales en cuanto al género. En este sentido es importante la transición al mercado de trabajo que intuyen. Hay quien piensa en una transición suave, marcada por la proximidad. Como señalan Horcas López, Bernad i Garcia y Martínez Morales (2015), son muchos los jóvenes que pasando por estos itinerarios de segunda oportunidad pretenden acceder a un mercado de trabajo protegido, como lo estaban en el centro educativo (salir sin salir), a través de sus redes de proximidad e incluso de la familia. Sin embargo, el mercado de trabajo no funciona con las mismas dinámicas protectoras que se ejercen en los centros educativos, lo que genera «una tensión entre la dinámica protegida del espacio educativo, y la exigencia del espacio sociolaboral» (Horcas López, Bernad i Garcia y Martínez Morales, 2015: 218).

En definitiva, las entrevistas realizadas a alumnado de FPB, con el objetivo de comprender la segregación por género en esta opción de estudios, evidencian la pervivencia de roles de género muy tradicionales, especialmente en el caso de los chicos. 


\section{CONCLUSIONES}

El objetivo del artículo ha sido aportar elementos que ayuden a comprender desde las narraciones de sus protagonistas la segregación por sexo que encontramos en las estadísticas acerca del acceso y la permanencia en los ciclos formativos de FPB. Todo ello a partir del concepto de itinerarios generizados. Para ello, nos basamos en datos cuantitativos de fuentes secundarias y en el estudio cualitativo realizado basado en entrevistas a alumnado de centros educativos valencianos.

El recorrido a través de las estadísticas educativas en FP muestra una persistencia de asimetrías de sexo que contribuyen a conformar lo que denominamos itinerarios generizados, un fenómeno que aparece intensificado en el caso de la FPB. Los chicos continúan concentrándose de manera clara en aquellos títulos de la FPB que se corresponden con sectores laborales masculinizados, como es el caso de Electrónica y Electricidad o Transporte y Mantenimiento de Vehículos, con porcentajes que superan el 9o \%. Y ellas, en el título de Peluquería y Estética, donde superan la proporción del $80 \%$ respectivamente.

Esta segregación horizontal de los estudios de FPB evidenciada por las cifras se traslada a las narrativas de las chicas y los chicos entrevistados, haciéndola más rica y superando la división por sexo que las estadísticas nos proporcionan. Los discursos del alumnado permiten identificar cómo se elaboran ideas y estereotipos de itinerarios generizados, con masculinidades y feminidades hegemónicas vinculadas. En las entrevistas realizadas se verbaliza una justificación básica en esta división de itinerarios: sus habilidades y preferencias, ellos más proclives a las habilidades manuales, a las maquinarias, y ellas más centradas en la imagen personal, en las relaciones personales e incluso en el cuidado. Una justificación que pivota en lo personal cuando tanto las palabras como las cifras son claras al indicar que hay un componente estructural que ha sido interiorizado en una socialización de género en la que sus iguales son un elemento fundamental. En este sentido, son frecuentes las referencias a los amigos y las amigas en la toma de decisiones ante su trayectoria formativa. Pero también son fundamentales las actitudes y las opiniones —o la expectativa de estas- del resto de personas de su misma edad con las que comparten espacios en el centro educativo o en su barrio y ciudad. En ambos casos son los chicos los que se muestran más atentos a posibles sanciones negativas ante decisiones o comportamientos que rebasen la norma social de género respecto a la elección de estudios. Se repliegan a la idea de masculinidad hegemónica. Sin embargo, parece como si las chicas fueran más permeables a los esfuerzos 
realizados en materia de igualdad de género en los centros y en la sociedad en general y muestran un discurso más igualitario, apelando a que hombres y mujeres están capacitados a realizar cualquier actividad de igual manera. Ahora bien, las prácticas nos muestran que no dejan de optar por itinerarios claramente feminizados, cumpliendo, pues, con el mandato de género, aunque sea disonante con sus palabras.

En este sentido, lo que denominamos itinerarios generizados se producen y se seguirán reproduciendo si no se promueve un análisis profundo de los factores que contribuyen a construir los itinerarios como generizados, porque, aunque disponemos de estadísticas desagregadas por sexo, no se está midiendo el peso de las escuelas como entornos e instituciones de género, así como el papel del profesorado en esa construcción cultural.

\section{BIBLIOGRAFÍA}

Acker, Joan (1990). Hierarchies, jobs, bodies: a theory of gendered organizations. Gender \& society, $4(2), \quad 139-158$. doi: 10.1177/089124390004002002

Alheit, Peter y Dausien, Bettina (2007). En el curso de la vida. Xàtiva: Instituto Paulo Freire, CREC.

Arnot, Madeleine (2002). Reproducing Gender? Essays on educational theory and feminist politics. New York: Routledge Falmer.

Barberà, Esther, Candela, Carlos y Ramos, Amparo (2008). Elección de carrera, desarrollo profesional y estereotipos de género. Revista de Psicología Social, 23 (2), 275-285. doi: 10.1174/021347408784135805

Cabrera, Leopoldo (1996). La explicación sociológica de la demanda y distribución del alumnado de Formación Profesional. Revista Española de Investigaciones Sociológicas, 76, 193-218. Recuperado de: http://www.reis.cis.es/

Connell, Raewyn (2001). Educando a los muchachos: la educación física mixta en clave de género. Nómadas, 14, 156-173. Recuperado de: $\quad$ http://nomadas.ucentral.edu.co/index.php/inicio/35construcciones-de-genero-y-cultura-escolar-nomadas-14?start $=12$ 
Eurostat (2018). Early leavers from education and training by sex. Recuperado de: https://ec.europa.eu/eurostat/web/productsdatasets/-/sdg_O4_10

Fernández, Ana Belén e Ibáñez Pascual, Marta (2018). Más mujeres en los estudios de Informática: una propuesta desde el departamento de formación y orientación laboral, Revista de Sociología de la Educación-RASE, 11(1), 116-134. doi: 10.7203/RASE.11.1.10624

Frutos, Lola (2010). Identidad de género en las trayectorias académicas y profesionales de las mujeres. Revista de Sociología de la EducaciónRASE, 3 (3). 336-356. Recuperado de: https://ojs.uv.es/index.php/RASE/article/view/8716

García De León, M. Antonia y García De Cortázar, Marisa (2001). Las académicas (Profesorado universitario y género). Madrid: Instituto de la Mujer.

Guil Bozal, Ana y Flecha García, Consuelo (2015). Universitarias en España: De los inicios a la actualidad. Revista Historia de la Educación Latinoamericana, 17 (24), 125-148.

González Ramos, Ana M. (2014). ¿Camuflaje o transformación? Estrategia profesional de las mujeres en carreras tecnológicas altamente masculinizadas. Educar, 50 (1), 187-205. doi: $10.5565 / \mathrm{rev} /$ educar.63o

Hardy, Vincent, Burchell, Brendan, Rubery, Jill y Smith, Mark (2015).

New method to understand occupational gender segregation in European labour markets. European Commission. doi: 10.2838/748887

Horcas López, Vicent, Bernad i Garcia, Joan Carles y Martínez Morales, Ignacio (2015). ¿Sueña la juventud vulnerable con trabajos precarios? La toma de decisiones en los itinerarios de (In/Ex)clusión educativa. Profesorado: Revista de currículum y formación del profesorado, 19 (3), 210-225.

Jackson, Carolyn (2006). 'Wild' girls? An exploration of 'ladette' cultures in secondary schools, Gender and Education, 18(4), 339360. doi: 10.1080/09540250600804966 
Jackson, Carolyn y Tinkler, Penny (2007). 'Ladettes' and 'Modern Girls': 'Troublesome' Young Femininities. The Sociological Review, 55(2), 251-272. doi: 10.1111/j.1467-954X.2007.00704.x

Julià, Albert (2016). Contexto escolar y desigualdad de género en el rendimiento de comprensión lectora. Revista Española de Investigaciones Sociológicas, 156, 41-58. doi: 10.5477/cis/reis.156.41

Martínez García, José Saturnino y Merino, Rafael (2011). Formación profesional y desigualdad de oportunidades educativas por clase social y género. Témpora, 14, 13-37.

Ministerio de Educación y Formación Profesional (2019). Estadísticas de la Educación. Nivel de formación, Formación permanente y Abandono: Explotación de las variables educativas de la Encuesta de Población Activa. Recuperado de: http://www.educacionyfp.gob.es/servicios-alciudadano/estadisticas/mercado-laboral/epa.html

Obiol, Sandra (2017). Anar a la universitat, una decisió només individual? El paper de la família. En Villar, Alícia (coord.). La decisió d'anar a la universitat. Processos d'orientació $i$ transicions ducatives en temps dincertesa (67-86). València: Tirant lo Blanch.

Subirats, Marina y Brullet, Cristina (1988). Rosa y azul. La transmisión de los géneros en la escuela mixta. Madrid: Instituto de la Mujer.

Subirats Martori, Marina (2016). De los dispositivos selectivos en la educación: el caso del sexismo. Revista de Sociología de la EducaciónRASE, 9 (1), 22-36. Recuperado de: https://ojs.uv.es/index.php/RASE/article/view/8401

Tarabini, Aina, Curran, Marta, Montes, Alejandro y Parcerisa, Lluis (2019). Can educational engagement prevent Early School Leaving? Unpacking the school's effect on educational success. Educational Studies, 45(2), 226-241. doi: $10.1080 / 03055698.2018 .1446327$

Vázquez-Cupeiro, S. (2015). Ciencia, estereotipos y género. Una revisión de los marcos explicativos. Convergencia: Revista de ciencias 
sociales, $22(68)$, 177-202. Recuperado de: http://www.redalyc.org/articulo.oa?id=10536227007

Vergés Bosch, Núria (2012). De la exclusión a la autoinclusión de las mujeres en las TIC. Motivaciones, posibilitadores y mecanismos de autoinclusión. Athenea Digital. Revista de pensamiento e investigación social, 12(3), 129-150. doi: 10.5565/rev/athenead/v12n3.1042

Villar, Alícia, Grau, Arantxa y Obiol, Sandra (2014). Educació i génere. Propostes per pensar i repensar els géneres. València: Tirant lo Blanch. Villar Aguilés, Alícia (coord.) (2017a). La decisió d'anar a la universitat. Processos d'orientació universitária $i$ transicions educatives en temps d'incertesa. València: Tirant lo Blanch.

Villar Aguilés, Alícia (2017b). La decisió d'anar a la universitat. Raons d'una decisió decidida. En Villar, Alícia (coord.) La decisió d'anar a la universitat. Processos d'orientació i transicions educatives en temps d'incertesa. València: Tirant lo Blanch. 